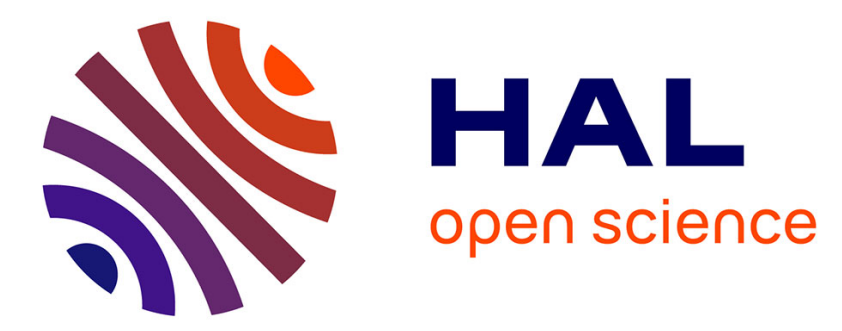

\title{
From flying wheel to square flow: Dynamics of a flow driven by acoustic forcing
}

Tristan Cambonie, Brahim Moudjed, Valéry Botton, Daniel Henry, Hamda Ben Hadid

\section{- To cite this version:}

Tristan Cambonie, Brahim Moudjed, Valéry Botton, Daniel Henry, Hamda Ben Hadid. From flying wheel to square flow: Dynamics of a flow driven by acoustic forcing. Physical Review Fluids, 2017, 2 (12), pp.123901. 10.1103/PhysRevFluids.2.123901 . hal-02946088

\section{HAL Id: hal-02946088 https://hal.science/hal-02946088}

Submitted on 22 Sep 2020

HAL is a multi-disciplinary open access archive for the deposit and dissemination of scientific research documents, whether they are published or not. The documents may come from teaching and research institutions in France or abroad, or from public or private research centers.
L'archive ouverte pluridisciplinaire HAL, est destinée au dépôt et à la diffusion de documents scientifiques de niveau recherche, publiés ou non, émanant des établissements d'enseignement et de recherche français ou étrangers, des laboratoires publics ou privés. 


\title{
From flying wheel to square flow: Dynamics of a flow driven by acoustic forcing
}

\author{
Tristan Cambonie, ${ }^{1, *}$ Brahim Moudjed, ${ }^{1}$ Valéry Botton,,${ }^{1,2, \dagger}$ Daniel Henry,,${ }^{1, \ddagger}$ \\ and Hamda Ben Hadid ${ }^{1}$ \\ ${ }^{1}$ Laboratoire de Mécanique des Fluides et d'Acoustique, CNRS, Université de Lyon, École Centrale de Lyon, \\ Université Lyon 1, INSA de Lyon, ECL, 36 Avenue Guy de Collongue, 69134 Ecully CEDEX, France \\ ${ }^{2}$ INSA Euro-Méditerranée, Université Euro-Méditerranéenne de Fès, Route de Meknès, BP51, Fez, Morocco
}

(Received 30 May 2017; published 8 December 2017)

\begin{abstract}
Acoustic streaming designates the ability to drive quasisteady flows by acoustic propagation in dissipative fluids and results from an acoustohydrodynamics coupling. It is a noninvasive way of putting a fluid into motion using the volumetric acoustic force and can be used for different applications such as mixing purposes. We present an experimental investigation of a kind of square flow driven by acoustic streaming, with the use of beam reflections, in a water tank. Time-resolved experiments using particle image velocimetry have been performed to investigate the velocity field in the reference plane of the experiments for six powers: $0.5,1,2,4,6$, and $8 \mathrm{~W}$. The evolution of the flow regime from almost steady to strongly unsteady states is characterized using different tools: the plot of time-averaged and instantaneous velocity fields, the calculation of presence density maps for vortex positions and for the maximal velocity and vorticity crest lines, and the use of spatiotemporal maps of the waving observed on the jets created by acoustic streaming. A transition is observed between two regimes at moderate and high acoustic forcing.
\end{abstract}

DOI: 10.1103/PhysRevFluids.2.123901

\section{INTRODUCTION}

Streaming phenomena associated with wave propagation in fluid media have been known for a long time [1-4]. One compulsory ingredient for these phenomena to be observed is dissipation, which can, depending on the configuration, be associated with viscous and thermodynamic effects in the bulk of the fluid or with boundary layer effects, in particular near walls. Here we are mainly interested in the case of a wave beam propagating within the fluid. We handle the particular case of ultrasound beams in liquids, but, interestingly, analogous phenomena can occur in situations involving laser beams and gravity wave beams [5], as recently observed in laboratory experiments [6,7]. Of course differences exist in the wave propagation properties and in the strength of the coupling, but strong similarities and common issues also exist, such as the capacity of the wave beam to reflect on boundaries of the fluid domain and the importance of mixing properties associated with the propagating beams. However, the case of streaming associated with acoustic beams has by far been the more studied case since celebrated pioneering works [8]. Ultrasonic acoustic streaming is in particular considered for its interest in a very broad variety of applications and processes, including acoustic velocimetry [9,10], sonochemistry applications [11-14], medical applications [15-18], acoustically assisted mixing, phase change, and blend separation [19-23], and metallic and semiconducting material processing [14,24-28]. In these applications, acoustic streaming is usually considered with an objective to better control the flow dynamics and/or improve its mixing properties. The possibility of acoustic streaming jets to reach a turbulence state $[8,14,29]$ and former stability studies [23,30-33] are further arguments in favor of this use.

\footnotetext{
*tristan.cambonie@gmail.com

${ }^{\dagger}$ valery.botton@insa.fr

†daniel.henry@ec-lyon.fr
} 


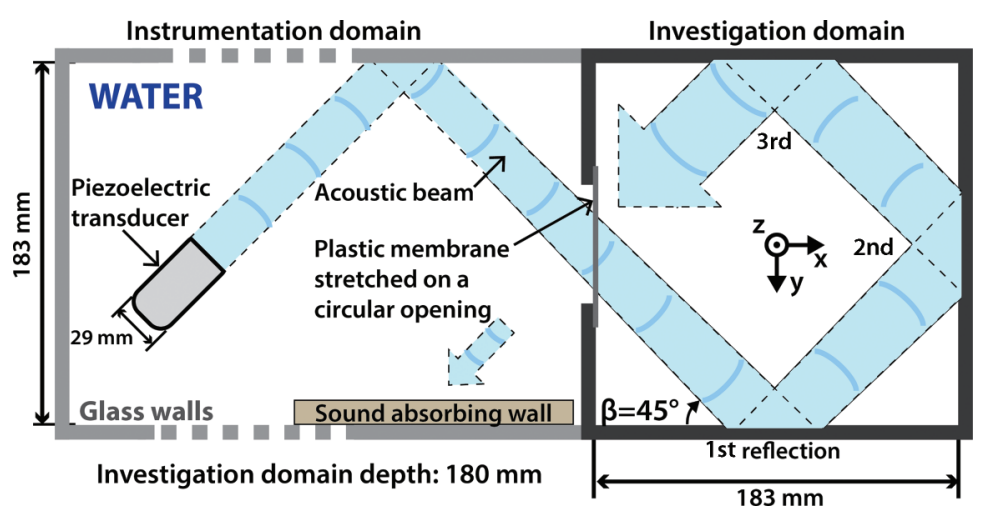

FIG. 1. Sketch of the experimental setup (top view at midheight of the domain). The origin of the Cartesian frame is set at the middle of the square investigation domain. The acoustic beam axis describes a square trajectory at midheight of this domain $(z=0 \mathrm{~mm})$ by reflecting at the center of each lateral glass wall. A glass lid on top of the cavity avoids the presence of a free surface.

In the studies associated with these applications, however, acoustic streaming flows are still very often seen as simple, straight, and steady flows, possibly after an initial transient of minor interest. In contrast to that, our former experimental observations have shown the existence of a ubiquitous and very rich dynamics [34,35], whose development is worth studying. These water experiments were made using ultrasounds in the megahertz range in several configurations [34,36-38].

In the following, we consider an experimental configuration in which an ultrasonic beam successively reflects on the walls of a square cavity so as to form a closed path. The resulting streaming flow is thus investigated as a function of the intensity of the acoustic forcing. This can be seen not only as a toy system for the investigation of acoustic streaming flow from an academic standpoint, but also as a way to approach an acoustic stirring system in an industrial container. The present investigation is fully experimental; however, some preliminary three-dimensional (3D) numerical computations using spectral element methods are also made. We will briefly refer to them to illustrate the case of low acoustic powers for which measurements are difficult since velocity levels are weak and relative experimental uncertainties become predominant. Based on particle image velocimetry (PIV) measurements, the evolution of the flow regime from almost steady to strongly unsteady states is considered using various tools: the plot of time-averaged and instantaneous velocity fields, the calculation of presence density maps for vortex positions and for the maximal velocity and vorticity crest lines, and the use of spatiotemporal maps of the waving observed on the acoustic jets.

The experimental setup is described in Sec. II. The three-dimensional topology of the timeaveraged velocity field is presented in Sec. III along with a few details on the numerical simulations. The results and a discussion will be presented in Sec. IV. In Sec. IV A, the evolution of the mean velocity field with the acoustic forcing is presented. In Sec. IV B, the flow topology and the instantaneous structures observed in the instantaneous velocity fields are discussed. In Sec. IV C, a transition of the flow topology between intermediate and high acoustic forcing is highlighted by looking at the vortices observed at the center of the measurement area. In Sec. IV D, the origin of the flow unsteadiness at intermediate acoustic forcing is studied. The characteristics of the flow unsteadiness at high acoustic forcing are developed in Sec. IV E. A summary is given in Sec. V.

\section{EXPERIMENTAL SETUP}

Experiments are performed in an $800 \times 183 \times 190 \mathrm{~mm}^{3}$ (length $\times$ width $\times$ depth) glass tank filled with de-ionized water. A top view of the setup is presented in Fig. 1. A glass wall separates the instrumentation domain, on the left-hand side of the picture, from the investigation domain, 
on the right-hand side. A glass lid is installed on top of the water to ensure no-slip boundary conditions and avoid free surface effects. The investigation volume is thus delimited by the glass lid, the glass separation wall, and the sidewalls and bottom walls of the glass tank. Its dimensions are $183 \times 183 \times 180 \mathrm{~mm}^{3}$. A 2-MHz circular plane piezoelectric transducer (from Imasonic ${ }^{\mathrm{TM}}$ with a diameter of $29 \mathrm{~mm}$ ) located in the instrumentation area is used to generate the acoustic beam. The separation wall is drilled with a $63-\mathrm{mm}$ hole covered with a thermoretractable plastic film to let the sound enter the investigation area and, at the same time, provide a rigid wall condition for the generated flow.

As depicted in Fig. 1, a $\beta=45^{\circ}$ tilt angle with respect to the $x$ axis is given to the transducer so that it creates a beam oriented towards the sidewall and located in the middle horizontal $z=0$ plane (namely, at $90 \mathrm{~mm}$ from the bottom and top walls). This acoustic beam first impinges at the glass sidewall in the instrumentation area, then reflects towards the center of the separation wall opening, and finally enters the investigation volume (Fig. 1) after a 280-mm travel distance. Because previous studies [34,36] carefully measured a 274-mm Fresnel length for this transducer, the acoustic field is known to have transited from the near field to the far field when it enters the investigation volume, making the jet flow structure simpler [37]. In the investigation domain, the acoustic beam impinges at the center of the first lateral glass wall with a $45^{\circ}$ angle and then successively on the second and third lateral glass walls before exiting the investigation area through the separation wall opening. Therefore, the acoustic beam axis describes a square path by successively reflecting at the center of each lateral wall. Because the incidence angle $\left(45^{\circ}\right)$ exceeds the critical angle $\theta_{c}=\arcsin \left(c_{1} / c_{2}\right)=16.2^{\circ}$, with $c_{1}$ and $c_{2}$ the respective velocities of sound in water and glass, the acoustic beam is therefore fully reflected in the direction of the center of the next wall. Finally, a sound absorbing plate made of Apflex F28 tiles from Precision Acoustics ${ }^{\mathrm{TM}}$ located on the acoustic beam path fully absorbs the acoustic beam in order to avoid any additional reflection.

A PIV system is used to measure the velocity field in the horizontal middle plane $(z=0)$ for the six electrical powers applied to the transducer: $P=0.5,1,2,4,6$, and $8 \mathrm{~W}$. It includes a continuous 532-nm Nd:YAG laser. Image acquisition is performed with a camera from Stemmer Imaging ${ }^{\mathrm{TM}}$ with a resolution of $2048 \times 2048$ pixels and with increasing frequencies of $2,2,4,5,5$, and $5 \mathrm{~Hz}$ associated, respectively, with the increasing electrical powers. In our measurements, 6000 double frames are acquired so that acquisition respectively lasted 50, 50, 25, 20, 20, and $20 \mathrm{~min}$. A 30-min pause is respected after any change of the electrical power in order to avoid any transient state and to make sure that only the steady state of the flow is observed. The de-ionized water was seeded with $5-\mu \mathrm{m}$ polyamid seeding particles of density $1030 \mathrm{~kg} \mathrm{~m}^{-3}$ from Dantec ${ }^{\mathrm{TM}}$. Note that, according to Ben Haj Slama et al. [39], only small seeding particles, like those used here, can reliably characterize flows induced by acoustic streaming. The temperature of water was measured to be $20.5^{\circ} \mathrm{C}, 20.7^{\circ} \mathrm{C}$, $20.7^{\circ} \mathrm{C}, 20.9^{\circ} \mathrm{C}, 21.2^{\circ} \mathrm{C}$, and $21.2^{\circ} \mathrm{C}$, respectively. Particle image velocimetry processing has been performed using $16 \times 16$ pixels interrogation windows and a $50 \%$ overlap using Davis ${ }^{\mathrm{TM}}$, Lavision's software.

A typical experimental time-averaged velocity field in the middle horizontal $z=0$ plane is presented in Fig. 2. The acoustic beam enters the measurement volume from the left at a $45^{\circ}$ downward angle and successively impinges at the center of each glass wall, describing a square loop in the investigation domain. Accordingly, the velocity field respects the square structure of the acoustic field and can therefore be decomposed in four similarly structured quadrants.

The fluid is accelerated along the acoustic beam trajectory forming four jets directly driven by the acoustic forcing (A in Fig. 2); we will refer to these jets as acoustic jets. Note that, due to the attenuation of the acoustic field by dissipation and the divergence of the acoustic beam, the acoustic intensity decreases along the beam path, so the successive acoustic jets have decreasing maximal velocities. Conversely to the totally reflecting acoustic beam, the acoustic jets do not reflect on the wall: The fluid entrained by the acoustic jets leaves the acoustic forcing areas and continues by inertia along the walls, forming what we will refer to as inertial jets (B in Fig. 2). At the center of the measurement domain, inside the square area formed by the acoustic jets, lies a wide recirculation area (C in Fig. 2), which is maintained in rotation by the action of the four acoustic jets. The last region 


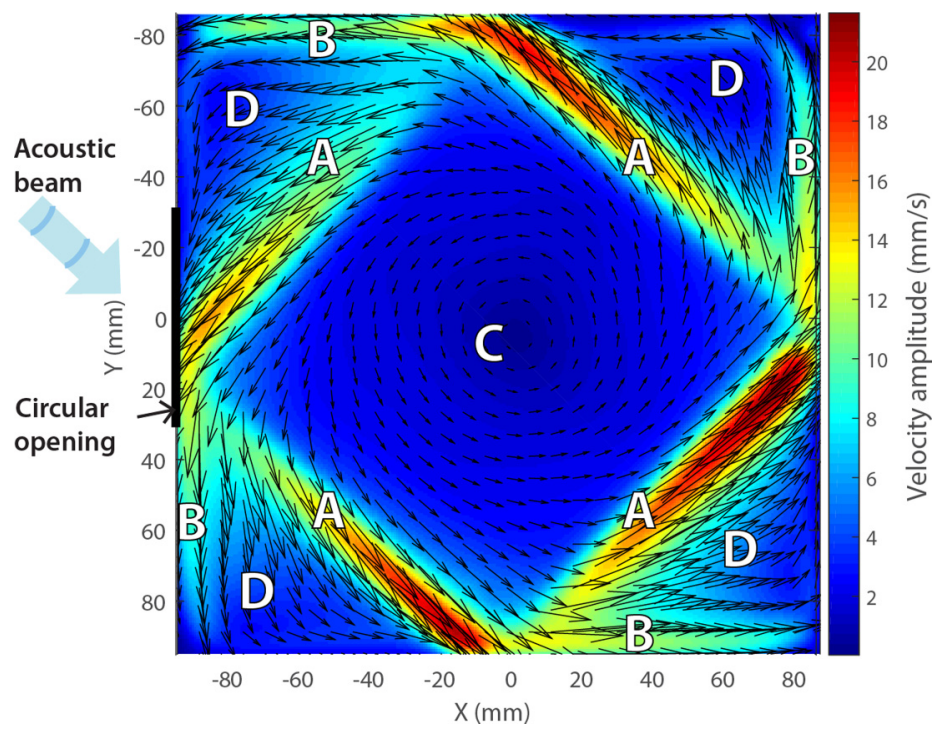

FIG. 2. Time-averaged velocity field and colored map of velocity magnitude obtained by $2 \mathrm{D}$ PIV in the $z=0$ horizontal plane of the investigation domain: A, acoustic jets; B, inertial jets; C, center area; and D, corner areas. The flow is generated by acoustic streaming at an electrical power of $P=6 \mathrm{~W}$. The acoustic beam enters the measurement volume from the left with a $45^{\circ}$ angle through a circular opening depicted as a thick black line. It then reflects successively on three sidewalls before leaving the measurement volume through the same opening following the path depicted in Fig. 1.

of interest is found in the corners of the measurement domain (D in Fig. 2), between the acoustic jets and the inertial jets, where an interaction between these two kinds of jets can be observed.

\section{NUMERICAL SIMULATIONS}

At this stage, it must be clarified that the considered configuration is not a $2 \mathrm{D}$ one. To get insight into the 3D structure of the flow, some preliminary numerical simulations have been performed. It is clear that the analysis of the complex dynamic behavior of such numerically simulated flow would necessitate a thorough bifurcation and instability study; however, we consider this study beyond the scope of the present paper.

The numerical simulations have been performed with a spectral element code, as described by Ben Hadid and Henry [40]. The time discretization, at third order, is carried out using a semi-implicit splitting scheme. The spatial discretization of the cavity is obtained with eight elements (two in each direction), each element being discretized with $31 \times 31 \times 21$ mesh points along the $x, y$, and $z$ directions, respectively. The driving force introduced in the Navier-Stokes equations is the volumetric acoustic force. It is proportional to the acoustic intensity, which is calculated at each mesh point following the Huygens-Fresnel assumption, as it is described in Refs. [38,40].

Figure 3 shows a 3D view of the steady velocity field obtained by numerical simulation for a $1-\mathrm{W}$ power, using an isosurface of velocity magnitude and 2D views in two particular planes: the $z=0$ symmetry plane and the $y=0$ plane. The symmetry plane corresponds to the plane in which the 2D PIV measurements took place. As expected, the arrow's orientation shows no out-of-plane component of the velocity, which confirms that the flow is two dimensional in this plane at low power. The isosurface displays the same principal features corresponding to the inertial and acoustic jets (here merged in a single isosurface due to the choice of the isosurface value). In the volume, the shape of the acoustic jets is a slightly expanding cylinder, because they keep the shape of the acoustic forcing, as formerly observed in a different configuration [36]. The inertial jets shape is 


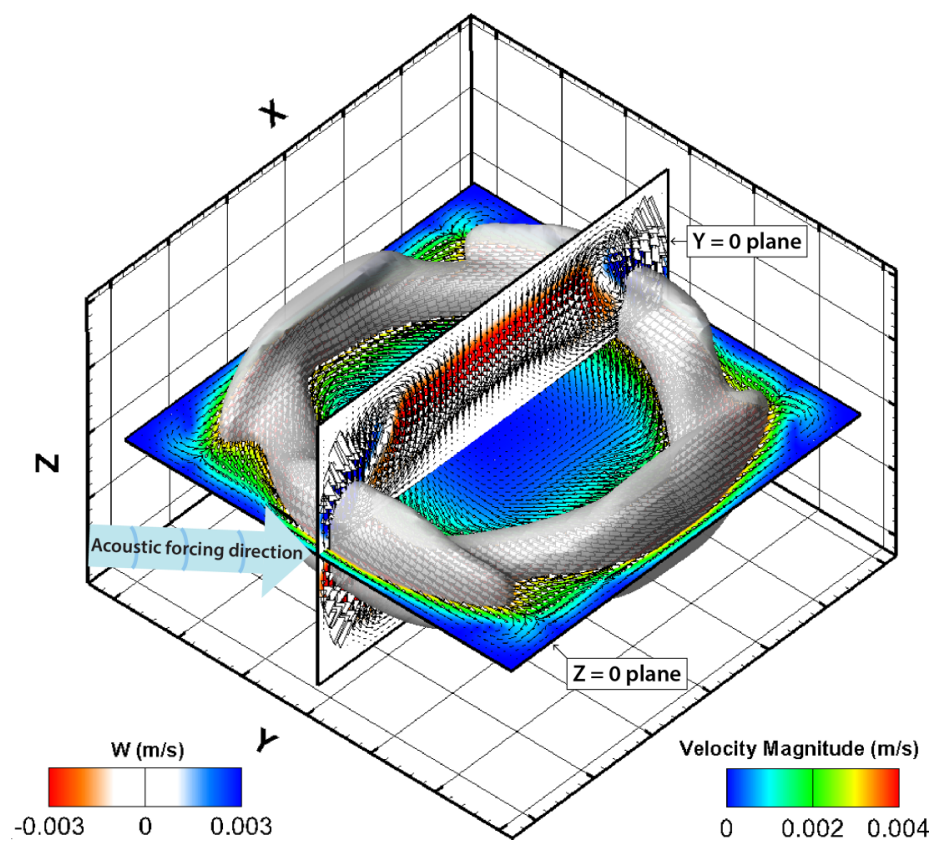

FIG. 3. Three-dimensional view of the steady velocity field obtained by numerical simulation for $1 \mathrm{~W}$ power, showing the $3.3-\mathrm{mm} \mathrm{s}^{-1}$ isosurface of velocity magnitude. The velocity field is also depicted in two planes: the symmetry plane $(z=0)$, with the colored map of velocity magnitude, and the $y=0$ plane, with the colored map of the vertical velocity $w$ to give indications on the velocity field outside the symmetry plane. For the vertical $y=0$ plane, only the in-plane velocity components ( $u$ and $w$, but not $v$ ) are given by the arrows to make the visualization of the vertical motion easier.

characteristic of an impinging wall jet, elongated along the wall in the direction of impingement, but also with global recirculations out of the symmetry plane, symmetrically on both sides of this plane. In the neighborhood of the impact points on the lateral walls, the fluid is then quickly driven away from the symmetry plane. Some additional particle tracking plots have shown that the fluid then recirculates at the top and bottom walls until it reaches the central zone of the cavity, where it moves vertically toward the symmetry plane and is eventually sucked in the acoustic jets by entrainment.

\section{RESULTS AND DISCUSSION}

\section{A. Time-averaged velocity fields}

Figure 4 shows the colored maps of the time-averaged velocity magnitude in the $z=0$ plane for increasing electrical powers $P$. Figures 4(a)-4(f) show the experimental velocity fields, while Figs. 4(g)-4(i) come from numerical simulations and focus on the very-low- to intermediate-acousticpower regimes. As can be seen from the color bar, the velocity level reached in the experiments is increased by roughly a factor 10, from 3 [Fig. 4(a)] to $30 \mathrm{~mm} / \mathrm{s}$ [Fig. 4(f)], when the power is increased from 0.5 to $8 \mathrm{~W}$. At low power $[P=0.5 \mathrm{~W}$, Fig. 4(a)], the velocity branches labeled as acoustic jets in Fig. 2 do not reach the walls and are deflected in the wall boundary layer by the pressure gradient. The square shape formed by the four acoustic jets presents then rounded corners in the vicinity of the reflection areas. In the low-power regime, this rounded structure is therefore quite different from the acoustic field structure, which exhibits a square shape whatever the power. The inertial jets are also not located close to the walls; they rather stay close to the newly generated acoustic jets, increasing the feeling of a circular circulation. Numerical simulations show that this phenomenon get still clearer at very low power where the flow adopts a seemingly flying 


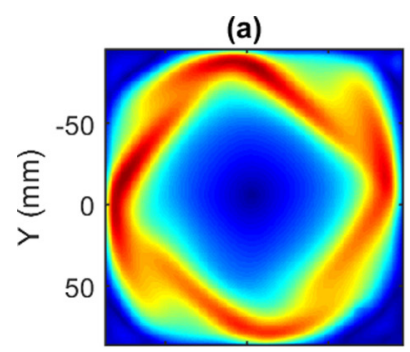

(c)

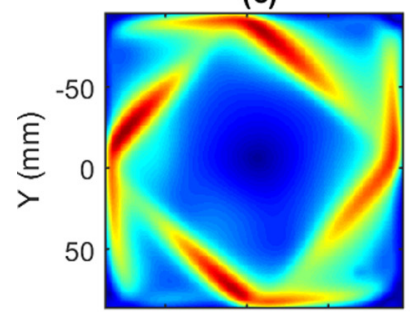

(e)

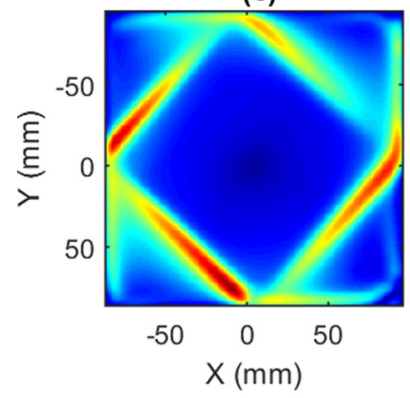

(g)
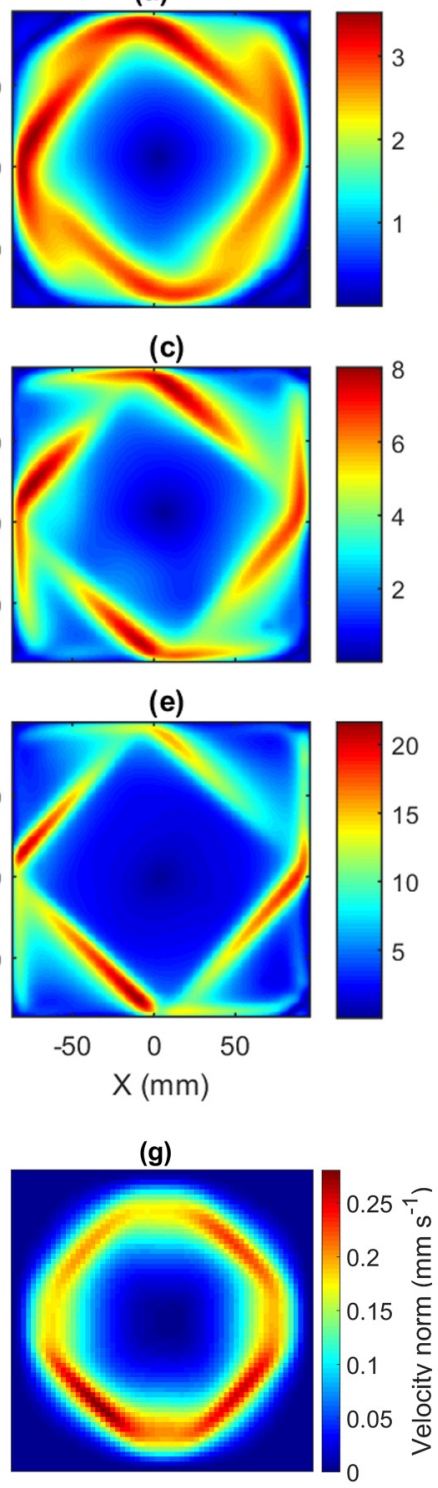

(b)

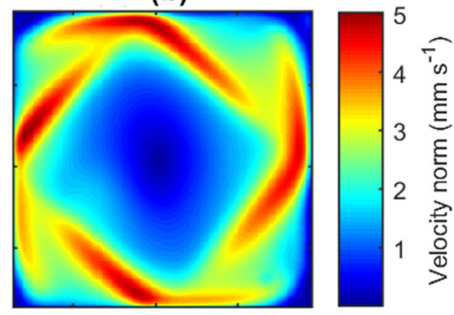

(d)

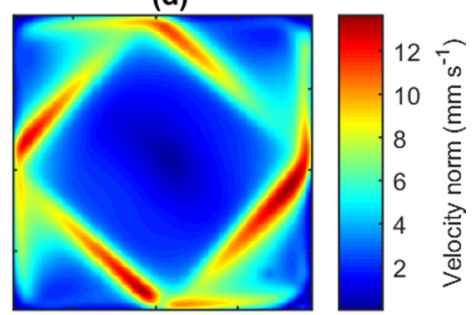

(f)

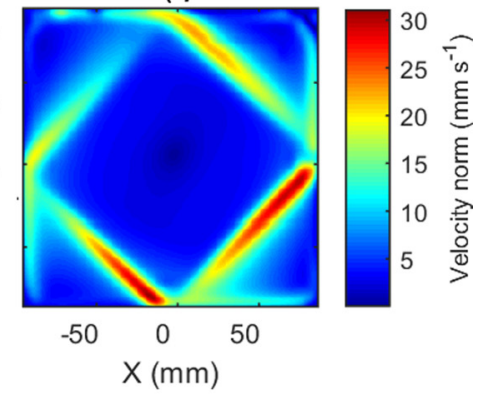

(h)

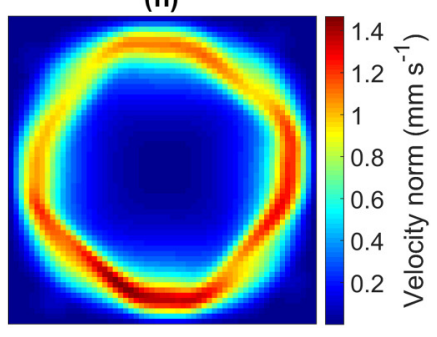

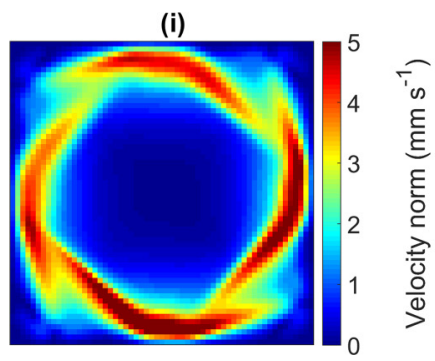

FIG. 4. Colored maps for the time-averaged velocity magnitude in the $z=0$ horizontal plane at different electrical powers $P$ : (a) $P=0.5 \mathrm{~W}$, (b) $P=1 \mathrm{~W}$, (c) $P=2 \mathrm{~W}$, (d) $P=4 \mathrm{~W}$, (e) $P=6 \mathrm{~W}$, (f) $P=8 \mathrm{~W}$, (g) $P=0.01 \mathrm{~W}$, (h) $P=0.1 \mathrm{~W}$, and (i) $P=1 \mathrm{~W}$. (a)-(f) Experimental results. (g)-(i) Numerical simulations. 
wheel circular structure and where the inertial jets are merged with the acoustic jets [Fig. 4(g)]. Figure 4 can now be used to describe the changes which affect the flow when the applied power is increased. Starting with a flying wheel structure, the inertial jets influence starts being visible in the numerics for $P=0.1 \mathrm{~W}[$ Fig. 4(h)] as a small deformation of the velocity isocontours at the base of the acoustic jets in the corner sides, and the inertial jets are finally formed and detached from the acoustic jets for $P=0.5 \mathrm{~W}$ [Fig. 4(a)]. It must also be said that the numerical solutions obtained at the three low acoustic powers corresponding to Figs. 4(g)-4(i) are found to be steady. At $P=1 \mathrm{~W}$, experimental results and numerical simulations [Figs. 4(b) and 4(i), respectively] show similar features. The inertial branches are longer, clearly distinct from the acoustic branches and closer to the walls. Beyond $P=1 \mathrm{~W}$ [Figs. 4(c)-4(f)], the inertial branches keep growing along the walls until they reach the next wall, where they take a $90^{\circ}$ turn and are redirected along this new wall. As expected from former observations [36], in this inertia-dominated regime, the acoustic jet's width and path match those of the acoustic beam. Let us recall that this was attributed to a longer momentum transverse diffusion time compared to the travel time along the jet. As a consequence, the higher the power is, the more pronounced the square shape in the flow pattern will be.

\section{B. Instantaneous velocity fields}

Figure 5 shows a time series of the instantaneous velocity magnitude for $P=8 \mathrm{~W}$, the most unsteady configuration. The structures and areas of interest already defined in Fig. 2 for the timeaveraged velocity field, i.e., acoustic and inertial jets, and corners and center areas, are also observed in the instantaneous velocity fields, but other phenomena appear, which can be viewed as specific features of the instantaneous dynamics. Online movies for each forcing power (movies Mov1.mp4 to Mov6.mp4 in Ref. [41]) prove very useful for apprehending the unsteady flow dynamics and the differences observed at low and at high acoustic forcing.

Lateral oscillations as well as velocity magnitude fluctuations affect both inertial and acoustic jets. These phenomena are observed over the entire range of investigated powers, from 0.5 to 8 W. The lateral waving of the acoustic jets has been emphasized by plotting the crest lines of the velocity isovalue maps in these areas as dashed lines. Nice examples of lateral oscillation are shown on the bottom right acoustic jet of Fig. 5(a) and on the top right acoustic jet of Fig. 5(b) (waving acoustic jets). The movies Mov1.mp4 to Mov6.mp4 in Ref. [41] are also appropriate to observe this phenomenon.

For the same top right acoustic branch, the velocity magnitude fluctuation can be clearly observed on the plots by looking either at the maximal velocity value or at the black $15-\mathrm{mm} \mathrm{s}^{-1}$-velocity isoline. In Figs. 5(c) and 5(d), a maximal velocity value around $16.5 \mathrm{~mm} \mathrm{~s}^{-1}$ is observed for this branch, considerably weaker than the $22-\mathrm{mm} \mathrm{s}^{-1}$ maximal value observed for the same branch a little later in Fig. 5(h). Movies labeled Mov7.mp4 to Mov12.mp4 are available in Ref. [41] and provide a relevant additional visualization of the velocity magnitude fluctuations observed along the velocity crest lines.

Both phenomena, the lateral waving of the acoustic jets and their maximal velocity magnitude fluctuations, have distinct dynamics. Indeed, strong waviness events can be observed without significant velocity fluctuations and, conversely, velocity fluctuations can happen without lateral waving of the acoustic jet.

In the central zone delimited by the four acoustic jets, the black $2-\mathrm{mm} \mathrm{s}^{-1}$-velocity isoline delimits multiple zones of velocity minima (central vortex), a main zone being located near the center of the domain and several smaller zones orbiting around it. While the main area of velocity minima is observed at all time steps, the amount of smaller orbiting areas can change with time. For instance, two such areas are observed in Fig. 5(a), zero in Fig. 5(b), and only one in Fig. 5(c). Using a vortex detection criterion $\left(\lambda_{C i}\right.$ criterion $\left.[42,43]\right)$ on the instantaneous velocity fields, we determined that these areas of velocity minima reveal the existence of instantaneous vortices in this central zone. We will return to these with more details in Sec. IV C. 
(a)
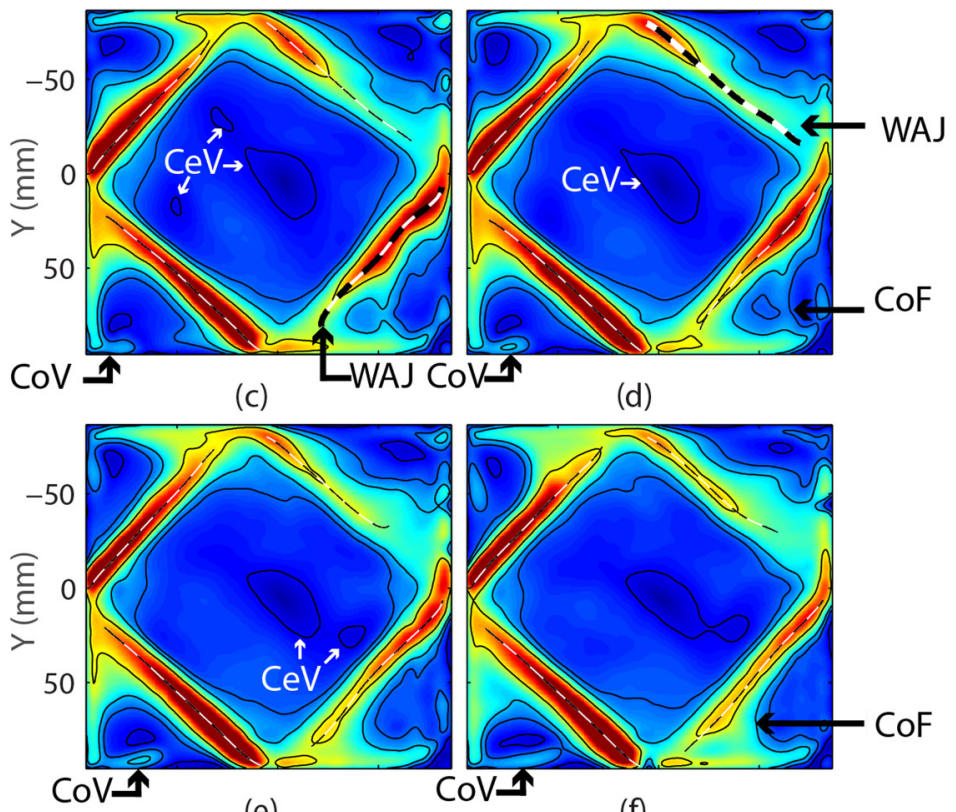

(e)

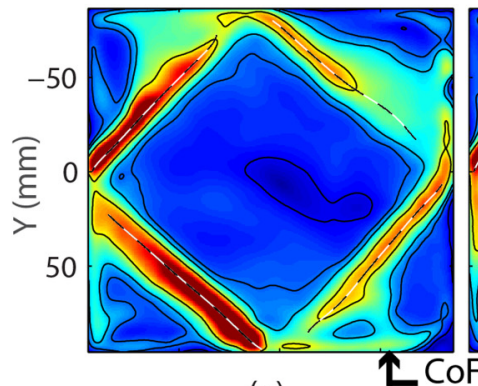

(g)
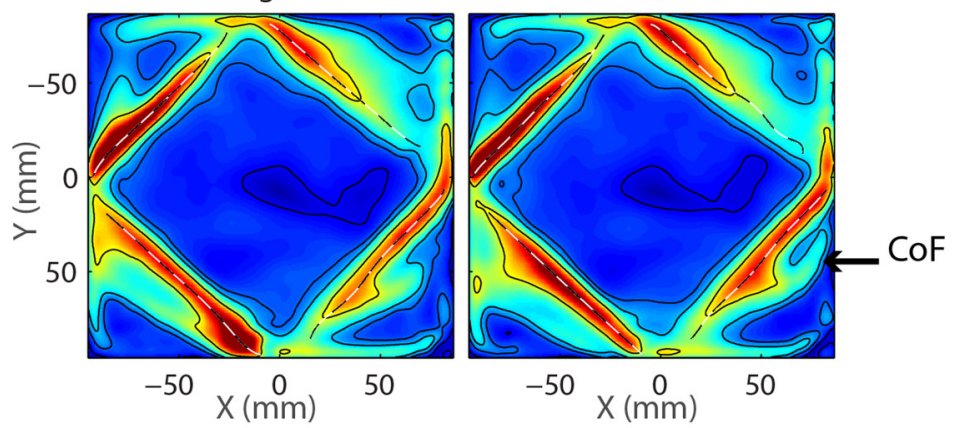

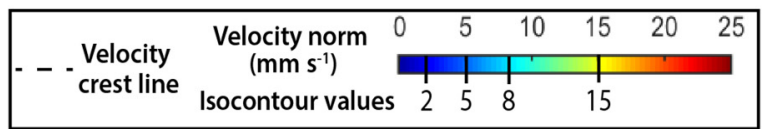

FIG. 5. Time series of the velocity magnitude colored map in the $z=0$ horizontal plane for the $P=8 \mathrm{~W}$ experiment: (a) $t=t_{\text {start }}$, (b) $t=t_{\text {start }}+2 \mathrm{~s}$, (c) $t=t_{\text {start }}+4 \mathrm{~s}$, (d) $t=t_{\text {start }}+6 \mathrm{~s}$, (e) $t=t_{\text {start }}+8 \mathrm{~s}$, (f) $t=t_{\text {start }}+10 \mathrm{~s}$, (g) $t=t_{\text {start }}+12 \mathrm{~s}$, and (h) $t=t_{\text {start }}+14 \mathrm{~s}$. Here $t_{\text {start }}$ is the beginning of the time series and not the beginning of the PIV recording. The following abbreviations are used: $\mathrm{CoV}$, corner vortex; $\mathrm{CeV}$, central vortex; WAJ, waving acoustic jet; and $\mathrm{CoF}$, corner filament. 
A strong unsteady dynamics is also observed between the inertial and acoustic jets for the strongest electrical powers $(P=4,6$, and $8 \mathrm{~W})$.

(i) Filaments of higher velocities (corner filaments) are created just downstream the impact area in the zone between the inertial and acoustic jets [see, for instance, the bottom right corner in Figs. 5(a) and 5(d)]. These filaments first connect the two jets. They are then transported in the corner zone by the jet-induced flow [Figs. 5(b) and 5(e)] and can finally disconnect from the jets [Figs. 5(f)-5(h)].

(ii) Counterclockwise vortices are periodically shed by the inertial jets in the corners (corner vortices). They travel along the wall from the corner to the next acoustic jet [see, for instance, the bottom left corner or the top left corner in Figs. 5(a)-5(d)]. As already stated, movies are available in Ref. [41] and can be very helpful for observing the specificity of this square flow unsteadiness and dynamics.

\section{Topological transition characterized by the central velocity minima}

In the top part of Fig. 6, density maps of the presence of the velocity minima in the central zone of the measurement domain have been obtained from the instantaneous velocity fields. A gray background is used when no minimum has been found over the 6000 PIV frames and the size of the small pixels gives a clear idea of the spatial resolution of the density maps.

These density maps are obtained by using the following procedure. First, detection of velocity minima is performed for all the instantaneous velocity fields at each power. Snapshots given in the bottom part of Fig. 6 for the $P=0.5,2,4$, and $8 \mathrm{~W}$ experiments show typical instantaneous velocity magnitude fields with red circles marking the velocity minima. Online movies (see movies Mov1.mp4 to Mov6.mp4 in Ref. [41]) illustrate the time evolution of the velocity minima.

Then the density maps are computed by concatenating all the velocity minima found over the 6000 PIV frames at each position in space and by expressing the total count of minima as a percentage. The steadiness of the minima over time, as well as the dispersion of the displacement of minima can thus be directly observed on these maps.

A transition is clearly observed between $P=2$ and $4 \mathrm{~W}$. Below $2 \mathrm{~W}$, the velocity minima are mainly contained in only one small area located at the center of the measurement domain. Direct observations of the velocity fields also show that only one minimum is seen in each frame. The density presence of this central minimum decreases while the power is increased from 0.5 to $1 \mathrm{~W}$. Surprisingly, this density increases at $P=2 \mathrm{~W}$, while the central minimum position is steadier. This fact will be discussed in Sec. IV D. Above $2 \mathrm{~W}$, a wide area is covered by the displacement of minima, still composed of a central area, now surrounded by a ring. The central area is more densely populated than the ring, but its extent is wider than for the smallest powers $(P \leqslant 2 \mathrm{~W})$ and the presence densities are one order of magnitude smaller. Direct observations of the velocity fields confirm these facts: A main central minimum is still observed in each frame, but its position is less stable than for smaller powers. Around this main central minimum revolve less marked minima entrained by the global in-plane recirculation of the flow generated by the acoustic jets.

The bottom plot of Fig. 6 shows the mean number of velocity minima per frame with respect to the power. As expected, only one minimum is observed below $2 \mathrm{~W}$. Above $2 \mathrm{~W}$, a regular increase of the minima per frame is observed, pointing out the progressive flow destabilization and the increasing complexity when the acoustic forcing is increased. In this range of powers, a close to linear relationship appears between the minima per frame and the acoustic forcing (electrical power). Since these velocity minima express the existence of vortices, the latter result can be seen as a topological transition occurring between 2 and $4 \mathrm{~W}$.

At low and intermediate acoustic forcing $(P \leqslant 2 \mathrm{~W})$, only one main vortex is found at the center of the measurement area, consistently with a regime where the overall instantaneous recirculation created by the acoustic jets is quasisteady and is very close to the time-averaged recirculation. In contrast, at high acoustic forcing, the existence of the other small orbiting vortices implies a more complex regime, which can no longer only be explained by a weakly unsteady global recirculation. Note that additional dynamic phenomena also appear in the corners at high acoustic forcing (lateral 
(a)

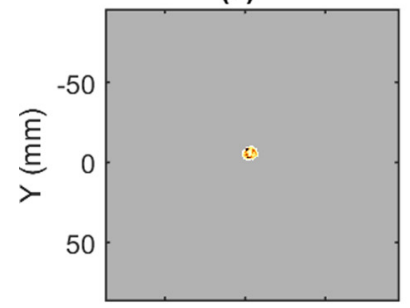

(c)

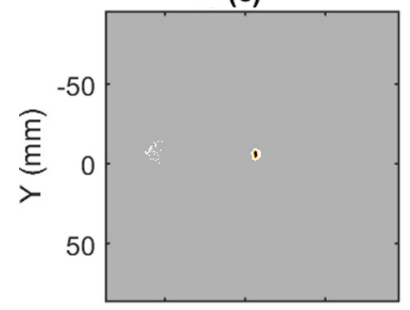

(e)

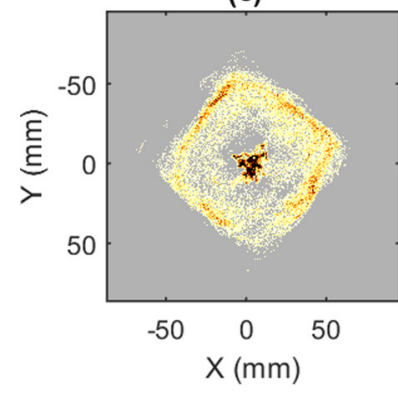

(b)
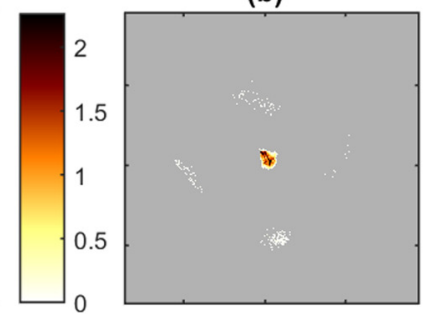

(d)
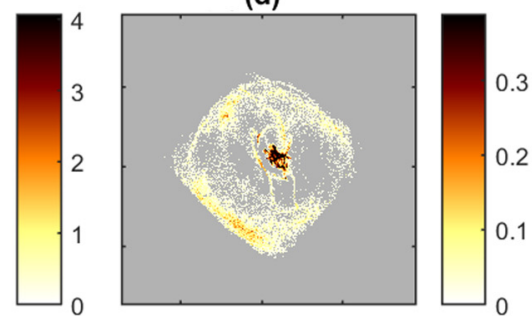

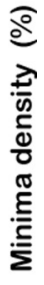

(f)

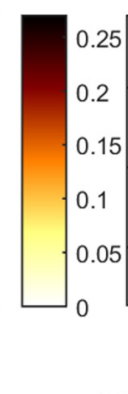

(g)

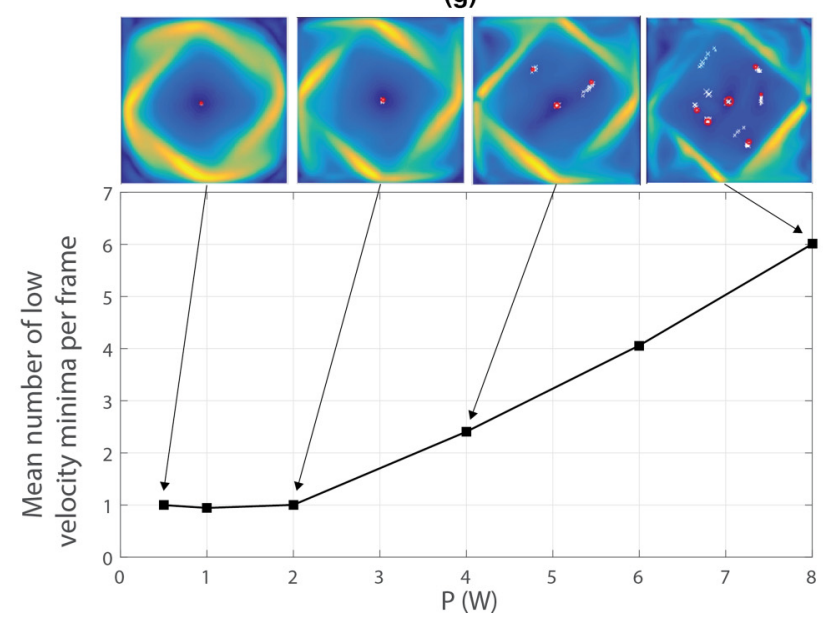

FIG. 6. (a)-(f) Density maps of the presence of velocity minima in the center of the measurement domain for the different electrical powers: (a) $P=0.5 \mathrm{~W}$, (b) $P=1 \mathrm{~W}$, (c) $P=2 \mathrm{~W}$, (d) $P=4 \mathrm{~W}$, (e) $P=6 \mathrm{~W}$, and (f) $P=8 \mathrm{~W}$. Gray color stands for no minimum found. (g) Graph of the mean number of velocity minima per PIV frame with respect to the power. Instantaneous velocity magnitude fields for the $P=0.5,2,4$, and $8 \mathrm{~W}$ experiments illustrate the position and the displacement of the detected minima. Red circles correspond to the velocity minima in the current frame, while the white crosses show the position of the velocity minima for the previous ten frames, as tails emphasizing the displacement of the minima (see [41] for a dynamic visualization). 
waving of the jets, corner vortices, velocity filaments, etc.). These phenomena indicate that the global flow becomes more complex. It is our idea that the increasing number of additional vortices in the central zone of the measurement domain can be seen as a good marker of this increasing complexity.

\section{Flow unsteadiness at intermediate acoustic forcing}

Direct observations of the experimental results (see the online movies in Ref. [41]) show a lateral waving of the inertial and acoustic jets. Such lateral waving has been characterized by studying the evolution of the instantaneous velocity crest lines of these jets (these crest lines are determined as the positions of the velocity magnitude maxima along these jets). Examples of instantaneous velocity crest lines for the acoustic jets are shown as dashed lines in Fig. 5.

Figures 7(a)-7(f) show spatiotemporal maps giving the local shift of the instantaneous crest positions with respect to the crest position for the time-averaged flow for the bottom left acoustic jet. Each column of a map gives this shift value along the crest curvilinear abscissa at a given time. Figures $7(\mathrm{~g})$ and $7(\mathrm{~h})$ illustrate how the shift is computed. A typical instantaneous velocity crest (green curve) is shown in Fig. 7(g), together with the mean velocity crest (black curve) extracted from the time-averaged velocity field. Curvilinear abscissas $S$ are defined along the mean crests and oriented with respect to the acoustic field propagation direction: $S=0 \mathrm{~mm}$ corresponds therefore to the beginning of the detected time-averaged velocity crest line, which characterizes the acoustic jet centerline. The shift between the instantaneous and time-averaged crest positions is defined by the distance between the curves, measured orthogonally to the mean curve. This shift is displayed in Fig. 7(h) as a function of the curvilinear abscissa $S$ along the mean crest line. By convention, this shift has been chosen to be positive (red colors) when the instantaneous crest is closer to the corner than the mean crest and negative (blue colors) when it is closer to the center. Figure 7 only shows the spatiotemporal maps of the bottom left acoustic jet, but such maps have been computed for each inertial and acoustic jet (cf. Fig. 11 to see the spatiotemporal maps of the four acoustic jets at $P=1$ and $8 \mathrm{~W}$ ). Note also that the same color bar is applied to the six spatiotemporal diagrams so that a glance at the intensity of the blue and red colors gives a good indication of the waving amplitude.

At intermediate acoustic forcing, very weak lateral waving of the acoustic jets is observed for the 0.5 - and 2-W configurations. At $P=0.5 \mathrm{~W}$, the waving evolves only slightly with time, the acoustic jet keeping almost the same deformation during long periods of time (several minutes). In fact, at this power, the velocity fluctuations are very weak and the flow can be considered as quasisteady. This is consistent with the numerical simulations, which have shown a steady behavior for such low acoustic powers as $P=0.01$ and $0.1 \mathrm{~W}$ [Figs. 4(g) and 4(h)]. Therefore, the weakness of the observed lateral waving of the acoustic jets at $P=0.5 \mathrm{~W}$ can be explained by the quasisteadiness of the flow. At $P=2 \mathrm{~W}$ [Fig. 7(c)], the waving is also weak, but alternations of red and blue regions indicate that it evolves regularly with time. At this power, the quasisteadiness of the flow cannot be used to justify this small oscillation amplitude, especially in regard to what is observed in the 1-W case. Indeed, the plot in Fig. 7(b) shows a very regular alternation of well marked and intense red and blue regions, indicating a strong and regular lateral waving of the acoustic jet. We observed about 18 occurrences of such events over $50 \mathrm{~min}$. Beginning from the end of the signal, the red and blue patterns can also be grouped by repeatable packets of three. From all this, it can be inferred that this case is quasiperiodic with a main frequency at about $6 \times 10^{-3} \mathrm{~Hz}$ and a smaller one-third harmonic. The more stable behavior of the 2-W configuration is also illustrated in Fig. 8, which shows density maps of the crests of velocity maxima found for the acoustic and inertial jets over the 6000 PIV frames available for each experiment. The situations at low acoustic forcing $(P=0.5,1$, and $2 \mathrm{~W})$ are displayed here, together with a situation at high acoustic forcing $(P=8 \mathrm{~W})$. The situations at $P=4$ and $6 \mathrm{~W}$ display features very similar to the $8-\mathrm{W}$ case.

The procedure used to obtain these velocity crest line density maps is similar to the one used for the density maps of velocity minima in Fig. 6: After a systematic detection of the velocity crest lines over the 6000 frames, all the velocity crest positions are concatenated at each spatial position and this crest presence count is finally expressed at each position as a percentage. 
(a)

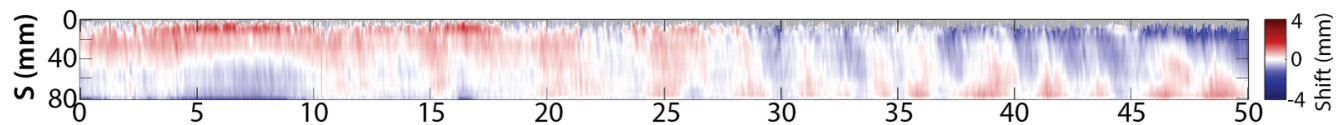

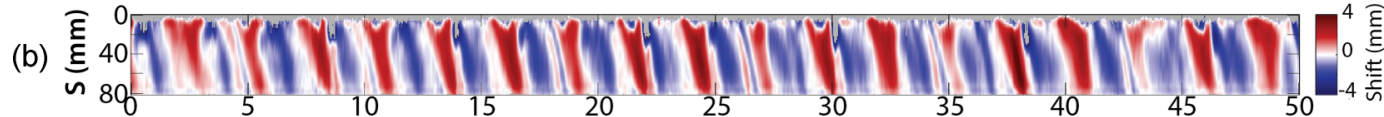

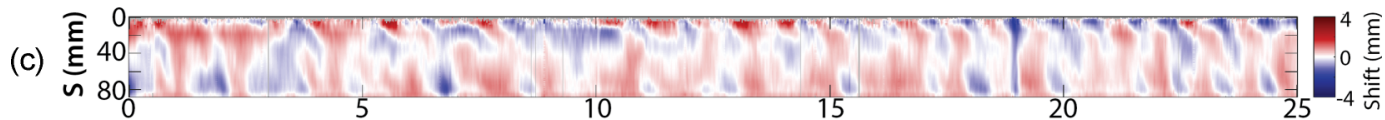
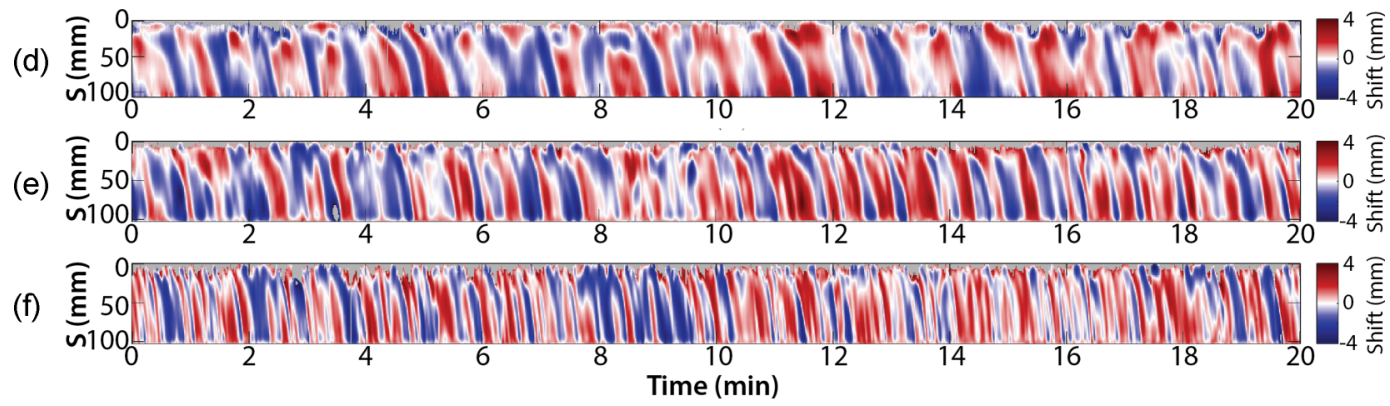

(g)

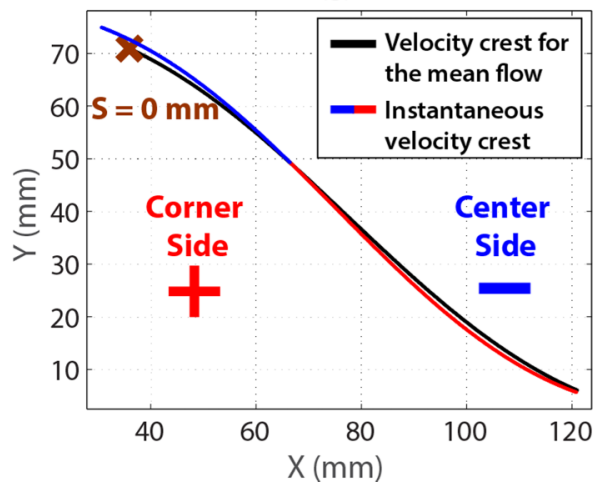

(h)

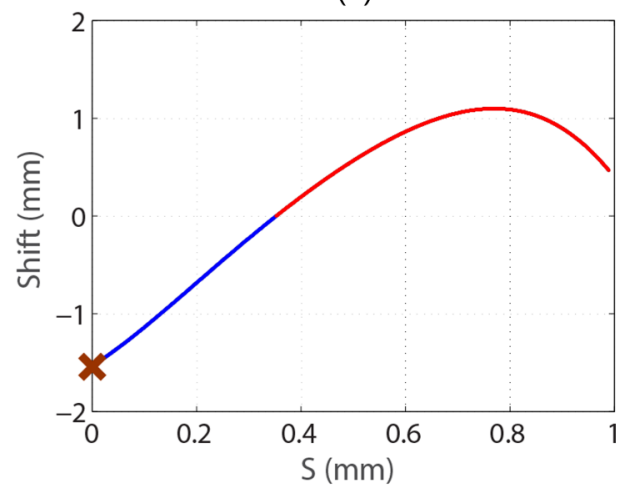

FIG. 7. (a)-(f) Spatiotemporal diagrams showing the velocity maximum crest shift with respect to the crest of the time-averaged flow for the bottom left acoustic jet and at different electrical powers $P:$ (a) $P=0.5 \mathrm{~W}$, (b) $P=1 \mathrm{~W}$, (c) $P=2 \mathrm{~W}$, (d) $P=4 \mathrm{~W}$, (e) $P=6 \mathrm{~W}$, and (f) $P=8 \mathrm{~W}$. The same color scale is chosen for all the cases. (g) Relative positions of the mean velocity crest extracted from the time-averaged velocity field and of an instantaneous velocity crest. (h) Shift of the instantaneous velocity crest shown in (g) with respect to the mean velocity crest. The shift is colored relative to its sign defined in $(\mathrm{g})$ : in red for an instantaneous positive shift toward the corner side and in blue for a negative shift toward the center side.

Both crest density values and lateral dispersion of the areas associated with a jet are therefore directly correlated with the lateral waving of this jet centerline. As expected, the crest density values are lower at high acoustic forcing than at intermediate acoustic forcing and crest presence areas are wider since the lateral waving of the jets is enhanced by the greater flow unsteadiness.

At low forcing, surprisingly, the crest density is maximum at $P=2 \mathrm{~W}$ and the crest presence areas of the jets are narrower than at $P=0.5$ and $1 \mathrm{~W}$. The inertial and acoustic jets of the 2-W experiment are therefore in more stable configurations than at $P=0.5$ and $1 \mathrm{~W}$.

This more stable behavior of the $2-\mathrm{W}$ experiment is likely linked to the relative positions of the inertial and acoustic jets. Indeed, in both the $0.5-\mathrm{W}$ and the $1-\mathrm{W}$ experiments, a correlation is observed between the proximity of the inertial and acoustic jets and their lateral dispersions. As a 
(a)

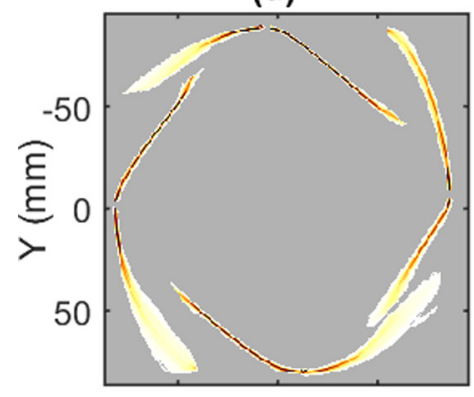

(c)

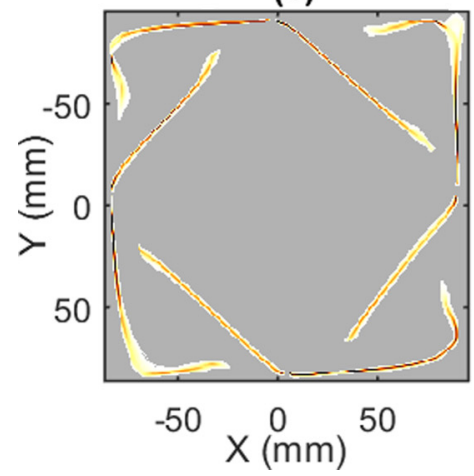

(b)
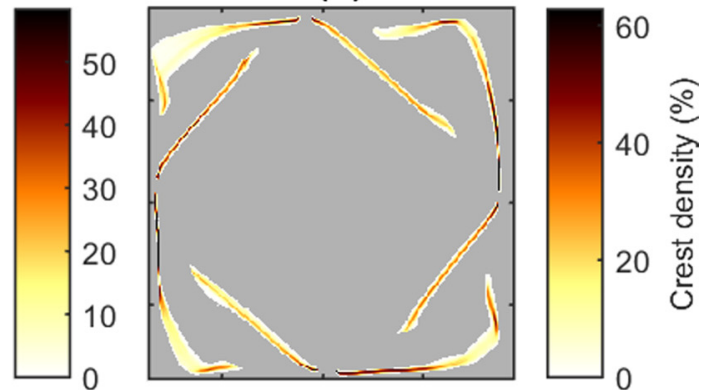

(d)

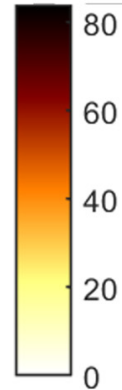

$$
0
$$

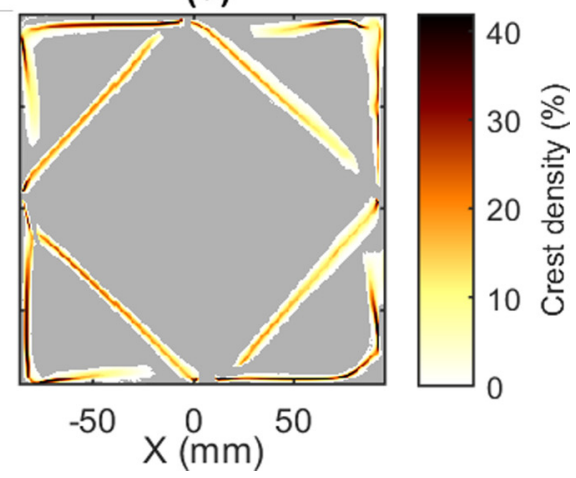

FIG. 8. Presence density maps of the crest lines of the velocity maxima associated with the inertial and acoustic jets for (a) $P=0.5 \mathrm{~W}$, (b) $P=1 \mathrm{~W}$, (c) $P=2 \mathrm{~W}$, and (d) $P=8 \mathrm{~W}$. Gray color stands for no crest found over the 6000 frames.

result, in the 2-W experiment where the inertial and acoustic jets are farther from each other, the jets are less able to influence and destabilize each other. On the contrary, in the 1-W case, the inertial and acoustic jets are close enough for a strong interaction to occur between them, leading to the regular and strong lateral waving displayed in Fig. 7(b).

Beyond $2 \mathrm{~W}$, a more complex instantaneous dynamics is observed in the corners (see Fig. 5) with filaments of high velocities connecting the inertial and acoustic jets and counterclockwise vortices. Independently of the distance between inertial and acoustic jets, the widening of the area covered by the velocity crests is not associated with the inertial and acoustic jets proximity, but is rather due to the presence of the corner instantaneous structures (even if, similarly to what is observed at low acoustic forcing, the inertial jets display stronger lateral waving at their ends when the inertial jets get closer to the acoustic jets). A partial conclusion, confirming the investigation of velocity minima in Sec. IV C, is that the $P=2 \mathrm{~W}$ case appears as an intermediate state between two different dynamical regimes.

\section{E. Flow unsteadiness at high acoustic forcing}

Figures 7(d)-7(f) depict the spatiotemporal maps of the 4-, 6-, and 8-W experiments. The high acoustic forcing cases show strong lateral waving of the acoustic jets, but without the quasiperiodic regularity observed in the $1-\mathrm{W}$ case. The waving events also occur at a considerably higher rate. Note that even if Figs. 7(b) and 7(d) seem to present similar periods of waving, the temporal scale used on the abscissa is quite different. In the 1-W experiment, 18 waving events happen over a 50-min-long experimental run, that is to say, about one event every $2 \min 45 \mathrm{~s}$, while in the 4-W experiment, a fast Fourier transform on the spatiotemporal map shows a main peak around 
TABLE I. Main event frequency $f$ and characteristic time $1 / f$ at $P=1,4,6$, and $8 \mathrm{~W}$ obtained from fast Fourier transforms performed on the spatiotemporal maps.

\begin{tabular}{lcc}
\hline \hline Forcing power $P(\mathrm{~W})$ & Frequency $f(\mathrm{~Hz})$ & Characteristic time $1 / f(\mathrm{~s})$ \\
\hline 1 & 0.0061 & 163.9 \\
4 & 0.023 & 43.5 \\
6 & 0.0355 & 28.2 \\
8 & 0.0494 & 20.25 \\
\hline \hline
\end{tabular}

$0.023 \mathrm{~Hz}$, indicating one event every $43.5 \mathrm{~s}$. This characteristic time for the occurrence of one event keeps decreasing for higher acoustic forcing (28.2 s in the 6-W case and $20.25 \mathrm{~s}$ in the 8-W case) as summarized in Table I.

In Fig. 9, the wavier cases of Fig. 7 are plotted again as dimensionless spatiotemporal diagrams; the considered powers are thus $1,4,6$, and $8 \mathrm{~W}$ for Figs. $9(\mathrm{a})-9(\mathrm{~d})$, respectively. The couple $(T, S)$ (where $T$ denotes time) gets rescaled into $\left(T \cdot V_{\max } / L_{\text {ac jet }}, S / L_{\text {ac jet }}\right)$, where $L_{\text {ac jet }}$ is the length of the acoustic jet and $V_{\max }$ is the maximal velocity on the acoustic jet. Further, $L_{\text {ac jet }}$ approximately keeps the same length between 80 and $100 \mathrm{~mm}$ (cf. Fig. 7), while the maximal velocity increases from 3 to $30 \mathrm{~mm} / \mathrm{s}$ when the power is increased from 0.5 to $8 \mathrm{~W}$.

A thick black dotted line emphasizes the slope corresponding to the waving events propagation along the curvilinear abscissa with an advection velocity matching the velocity of the acoustic jet (in the rescaled axes). It shows a similar scaling relationship of the waving event convection with the maximal velocity along the acoustic jet. Nevertheless, two slightly different slopes can be discerned:

(a)

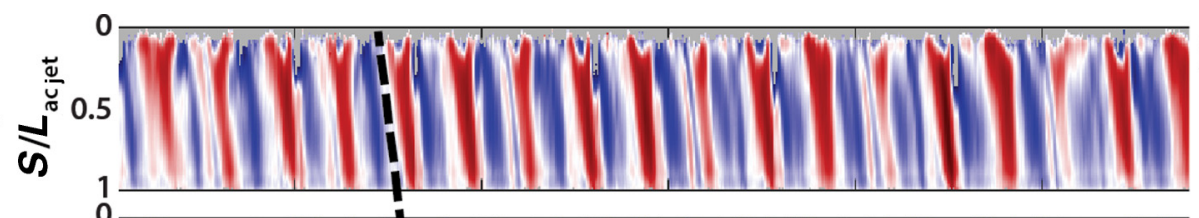

(b)

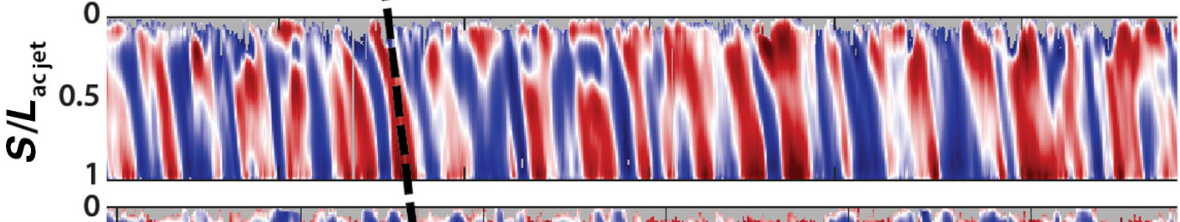

(c)

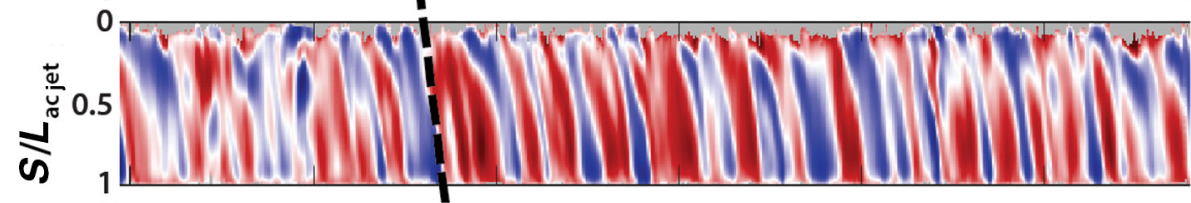

(d)
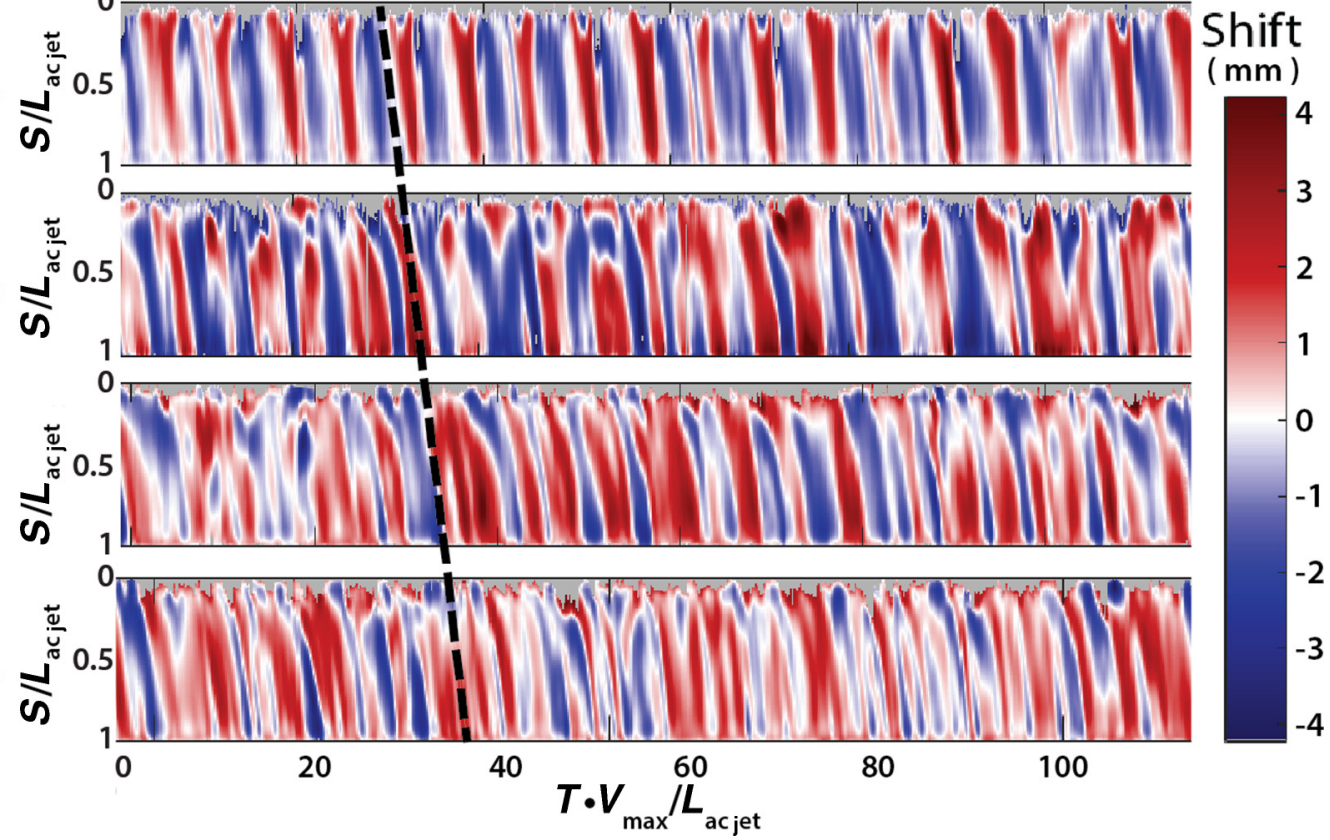

FIG. 9. Normalized spatiotemporal maps of the shift of the velocity maxima crest lines: (a) $P=1 \mathrm{~W}$, (b) $P=4 \mathrm{~W}$, (c) $P=6 \mathrm{~W}$, and (d) $P=8 \mathrm{~W}$. The curvilinear abscissa is normalized by the length of the acoustic jet $L_{\text {ac jet }}$ and the time by $L_{\text {ac jet }} / V_{\max }$, where $V_{\max }$ is the maximal velocity on the acoustic jet. 

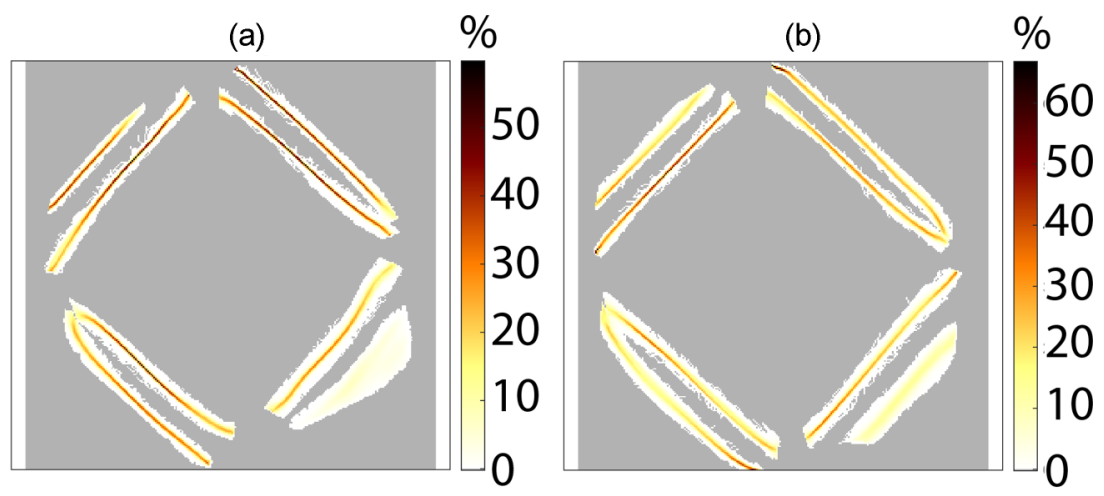

(c) $\%$
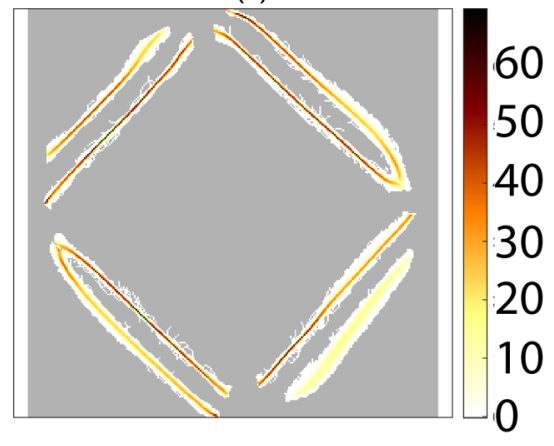

(d)
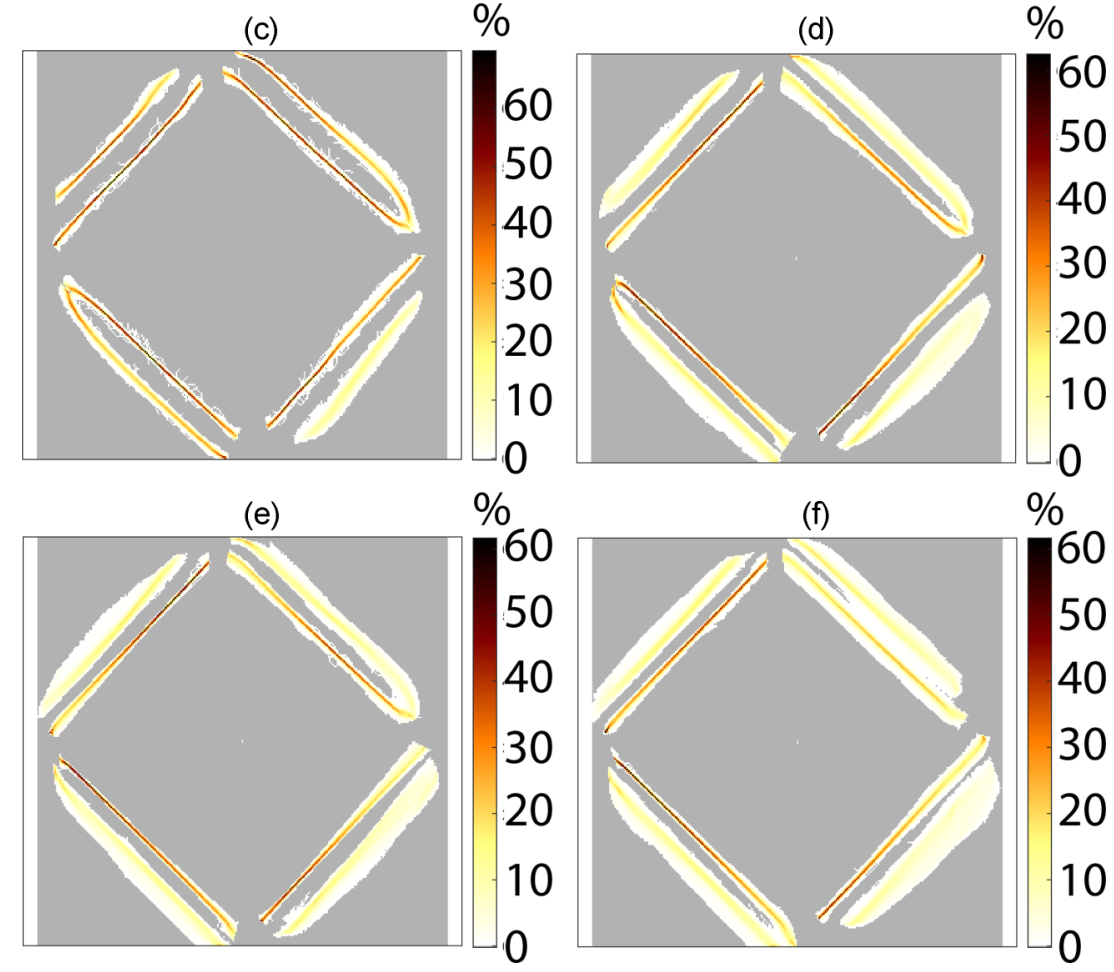

FIG. 10. Presence density maps of the crest lines of the vorticity maxima associated with the acoustic jets: (a) $P=0.5 \mathrm{~W}$, (b) $P=1 \mathrm{~W}$, (c) $P=2 \mathrm{~W}$, (d) $P=4 \mathrm{~W}$, (e) $P=6 \mathrm{~W}$, and (f) $P=8 \mathrm{~W}$. Gray color stands for no crest found over the 6000 frames.

one for the 1-W case below the already mentioned transition in Sec. IV C and another slightly lower for the high acoustic forcing cases.

Consistently with the flow complexification observed in Fig. 6 on the vortices present in the flow center, a complexification of the flow dynamics can be observed on the vorticity in the acoustic jet zones. This is well illustrated in Fig. 10, where presence density maps of the crest lines of the vorticity maxima associated with the shear layers on both sides of the acoustic jets are drawn. Here also the dispersion of the maximal vorticity crest lines is directly linked for each acoustic jet with the lateral waving of the corresponding corner and center shear layers.

In Fig. 10, quite similar dispersions of the corner and center maximal vorticity crest lines are observed at intermediate acoustic forcing [Figs. 10(a)-10(c)], characteristic of similarly affected 
(a)

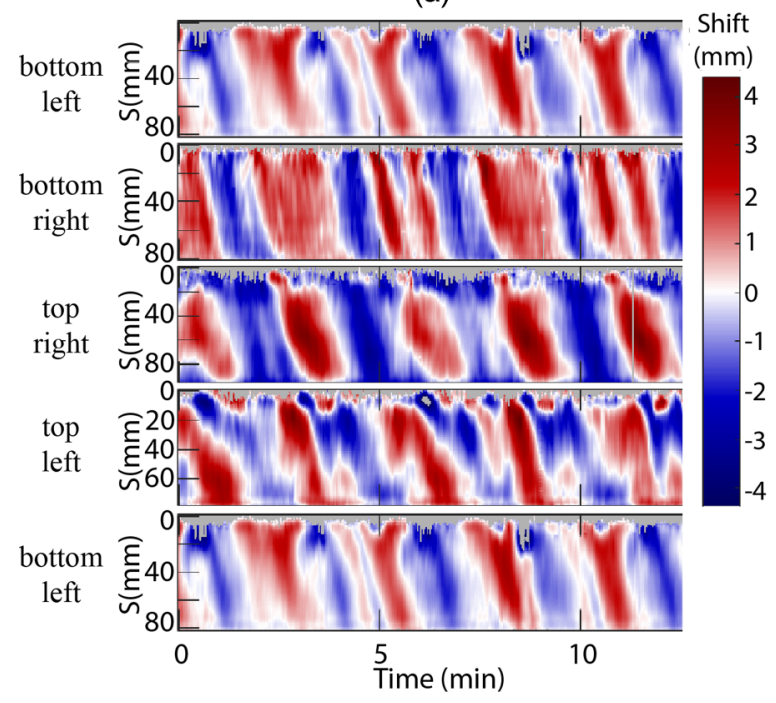

(b)

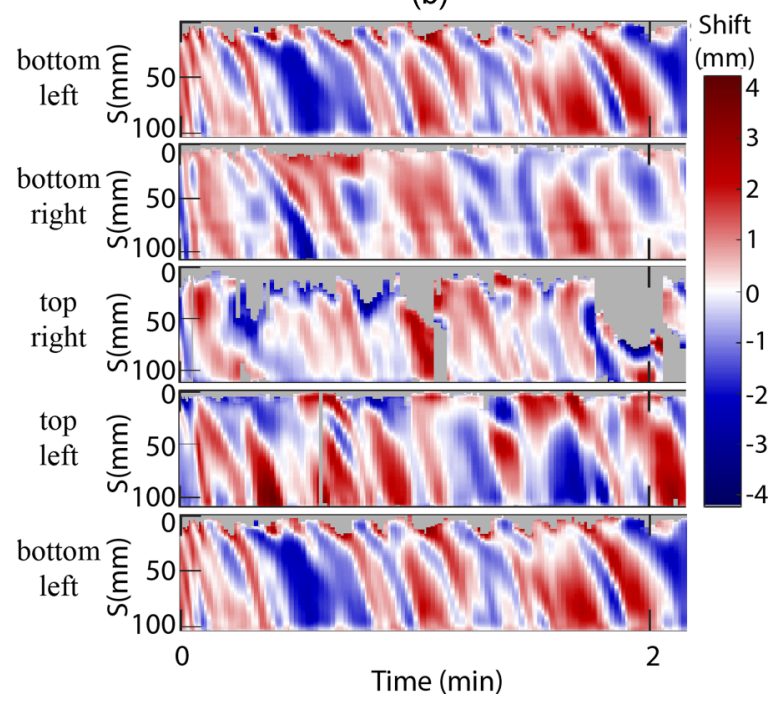

FIG. 11. Close-up of the spatiotemporal maps showing the shift of the crest lines of the velocity maxima: (a) $P=1 \mathrm{~W}$ and (b) $P=8 \mathrm{~W}$. The four acoustic jets are considered, as they appear along the acoustic beam; hence, shown, from top to bottom, are the bottom left, the bottom right, the top right, the top left, and again the bottom left acoustic jets. Gray color stands for no crest found at a given space-time location.

shear layers on both sides of the jet. In contrast, above $P=2 \mathrm{~W}$, an asymmetric dispersion is observed: The dispersion of the crest lines of the corner side shear layer is more spatially extended, characteristic of larger perturbations in the shear layer on the corner side than on the center side [Figs. 10(d)-10(f)].

Finally, in order to illustrate another important feature of the intermediate to high acoustic forcing transition, Fig. 11 gives a focus on a few minutes of the spatiotemporal diagrams of Fig. 9 in the 1-W and 8-W experimental runs. In contrast with Figs. 7 and 9, the four acoustic jets of each configuration are considered here, which will make it possible to estimate the synchronicity of the 
FROM FLYING WHEEL TO SQUARE FLOW: DYNAMICS OF ...

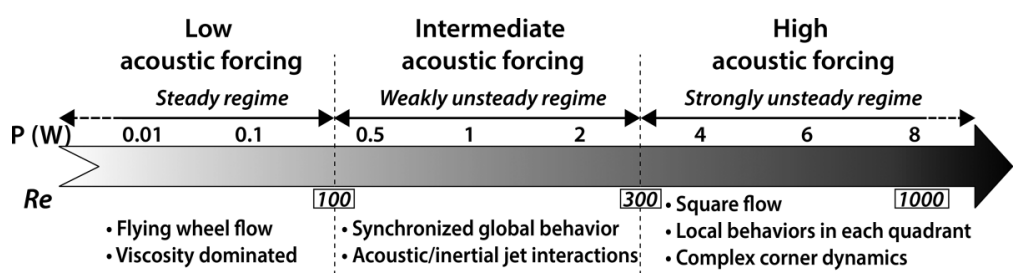

FIG. 12. Graph summarizing the changes of flow regime with increasing acoustic forcing.

acoustic jets waving. Consistently with the direct observations of the online movies, Fig. 11(a) shows that, at low and intermediate acoustic forcing, the waving of the acoustic jets is a rather global phenomenon, which occurs on the four acoustic jets almost simultaneously, in any case with a strong correlation. This behavior is lost at high acoustic forcing where each acoustic jet has its own dynamics, disconnected from the other jets' dynamics [Fig. 11(b)].

It should be noted that the acoustic jet instabilities involved in this unsteady flow dynamics are not related to the classical instabilities of the unforced free jet in its transition to a turbulent state. In the early stage of this transition, instabilities occur along the jet body as ring-shaped structures due to Kelvin-Helmholtz instabilities. Smaller vertical structures then appear when the ring-shaped structures are destabilized and break up, leading to the loss of coherence of the jet and to the transition to turbulence. This whole process is very sensitive to the Reynolds number and usually occurs over a rather short distance. The dynamics of the acoustic jet differ on many of these aspects.

(i) Concerning the Reynolds numbers involved in our acoustically driven flows (estimated as $\operatorname{Re}=U_{\max } \phi / \nu$, based on the maximal jet velocity $U_{\max }$ and the jet diameter approximated by the transducer diameter $\phi$ ), they are given in Fig. 12 for each flow transition, together with the different observed flow structures and regimes and the corresponding approximate power ranges. We see that the steady state of the jet occurs up to $\mathrm{Re}=100$ and the strongly unsteady regime (still far from turbulent) is only reached above $\operatorname{Re}=300$. In contrast, the transition to turbulence of the free jet is supposed to begin for $10<\operatorname{Re}<30$ [44], i.e., for smaller Re values.

(ii) Unlike the classical free jet, the acoustic jet body remains coherent and does not break up into small structures.

(iii) The acoustic jet instabilities exhibit different characteristics on both sides of the jet in the observed PIV plane: different spectra, amplitudes, wave velocities, with the inner side of the acoustic jets less destabilized than the side close to the wall (the reader can refer to the direct observation of the online movies). For example, this dissymmetry of behavior excludes ringlike instabilities for these acoustic jets.

Finally, other studies in less confined situations confirm these observations, i.e., a nonturbulent acoustic jet over very long distances of several decimeters in water [35] and even several meters in air [45], at already high-Reynolds-number values of 2200 [35] and 2000 [45]. All these arguments can convince us that the instabilities displayed by the acoustically forced jet are unrelated to the natural instabilities occurring on the classical free jet. These latter instabilities are unable to develop due to the extremely stabilizing effect of the acoustic forcing. Indeed, previous studies [34,36-38] showed that the acoustic jet local expansion quite accurately follows the width of the acoustic beam. The acoustic field has therefore a forcing effect as well as a confining effect, which both contribute to the stabilization of the acoustic jet compared to a free jet. In the present case of this square flow, the instabilities seem to be connected with the confinement of the flow by the square cavity: In addition to the acoustic jets, different flow structures (inertial jets, recirculation loops in the corners and in the core of the cavity, smaller corner and central vortices, etc.) are created, which interact with the acoustic jet and locally destabilize it, but without breaking it up and triggering a generalized turbulent state. 


\section{CONCLUSION}

We have presented an experimental investigation of a water-tank flow driven by acoustic streaming. Acoustic streaming is a noninvasive way of putting a fluid into motion using volumetric acoustic force developing all along the acoustic beam, in the core of the fluid domain. It is therefore considered as an interesting alternative to rotating mechanical stirring systems [46] which are very invasive and to electromagnetic stirring systems in which the developed Laplace forces penetrate the fluid volume by magnetic diffusion effects (thus sometimes subjected to limiting skin effects [47]).

Time-resolved experiments using particle image velocimetry have been performed to investigate the velocity field generated by a $2-\mathrm{MHz}, 29-\mathrm{mm}$ plane source fed with six different electrical powers: $0.5,1,2,4,6$, and $8 \mathrm{~W}$. The difference in the considered configuration is that the acoustic beam enters the tank horizontally at the center of a lateral face with a $45^{\circ}$ angle with respect to the tank wall, impacts the other tank glass walls with the same angle, and totally reflects on them. As a result, the acoustic beam describes a square path in the plane at midheight of the tank. The flow therefore exhibits a different pattern composed of four quadrants in which two jetlike structures are observed: an acoustic jet, directly driven by streaming along the acoustic beam, and an inertial jet, formed by inertia after the impact of an acoustic jet on a lateral wall.

Figure 12 sums up the different observed flow structures and regimes and the corresponding approximate power ranges.

(i) At very low and low acoustic forcing, the flow is steady. The very weak acoustic forcing is enough for the accumulation of momentum along the beams to create a global recirculation in the tank. In this regime, the acoustic jets adopt a round shape and do not follow the square path of the reflecting acoustic beam. In this regime the inertial and acoustic jets are therefore combined, forming a unique structure that we have denominated a flying wheel.

(ii) At intermediate acoustic forcing, the flow exhibits a weakly unsteady behavior, with lateral waving of the acoustic and inertial jets, which are now distinct and can interact with each other. Due to the acoustic forcing, the acoustic jet centerline position stays close to its time-averaged position (as seen in Fig. 8), while the inertial jet exhibits a wider waving, particularly at its end. This evolution of the flow is rather a global phenomenon with, for instance, a synchronization of waving of the four acoustic jets. Finally, even if the flow is not steady anymore, the observed flow structures in the instantaneous velocity field are nearly the same as those of the time-averaged velocity field.

(iii) At high acoustic forcing, the flow becomes strongly unsteady and follows closely the square shape of the acoustic beam path. The inertial jets stay very close to the lateral walls, creating a strong current in the vicinity of the lateral walls towards the corners. When their inertia is strong enough, the inertial jets reach the corners and even flow along the adjacent wall after a $90^{\circ}$ deviation, therefore locally adopting a typical $\mathrm{L}$ shape.

The acoustic and inertial jets waving in each quadrant are not as strongly correlated with their counterparts in the other quadrants. The flow dynamics is therefore no longer global. The waving of the jets and the fluctuations of the velocity composing the jets intensify with the acoustic forcing, indicating once again a complexification of the flow dynamics with the acoustic forcing. The jet waving and the associated velocity fluctuations are unrelated to a potential scenario of transition toward a turbulent state, but are rather linked to the interactions of the different flow structures generated in such a confined square cavity.

The instantaneous velocity fields strongly differ from the time-averaged velocity fields. Multiple instantaneous flow structures are observed.

(a) In the center of the measurement domain, several small vortices orbit the main central vortex, indicating changes in the global recirculation in the tank and a progressive complexification of the instantaneous flow topology.

(b) In the corners, filaments of velocity are generated between the acoustic and inertial jets. They initially connect the acoustic and inertial jets. They are then convected by the flow sweeping the tank corner and are elongated in the process until they detach. 
(c) Vortices are created due to the interaction of the inertial jets and the tank corners and are then convected along the tank wall, with a potential mixing effect in the boundary layers.

All these features appear as very interesting from the perspective of an efficient noninvasive mixing generated by acoustic streaming in a tank. In a square tank, while the tank corners usually are dead zones for mechanical stirring techniques, this study shows that acoustic streaming methods are able to generate flows in the vicinity of the tank walls and complex unsteady dynamics in the tank corners. From a more academic standpoint, the presently studied system can also be seen as an interesting well controlled system, with spatially nonhomogeneous forcing along rays, exhibiting very rich dynamics.

\section{ACKNOWLEDGMENT}

The authors acknowledge financial support from the Carnot institute Ingénierie@Lyon and from ARC Energies Région Rhône-Alpes in the form of a doctoral fellowship (B.M.).

[1] S. Boluriaan and P. J. Morris, Acoustic streaming: From Rayleigh to today, Int. J. Aeroacoust. 2, 255 (2003).

[2] M. Faraday, On a peculiar class of acoustical figures; and on certain forms assumed by groups of particles upon vibrating elastic surfaces, Philos. Trans. R. Soc. London 121, 299 (1831).

[3] Lord Rayleigh, On the circulation of air observed in Kundt's tubes, and on some allied acoustical problems, Philos. Trans. R. Soc. London 175, 1 (1884).

[4] N. Riley, Steady streaming, Annu. Rev. Fluid Mech. 33, 43 (2001).

[5] T. Dauxois, S. Joubaud, P. Odier, and A. Venaille, Instabilities of internal gravity wave beams, Ann. Rev. Fluid Mech., doi:10.1146/annurev-fluid-122316-044539.

[6] G. Bordes, A. Venaille, S. Joubaud, P. Odier, and T. Dauxois, Experimental observation of a strong mean flow induced by internal gravity waves, Phys. Fluids 24, 086602 (2012).

[7] H. Chraibi, R. Wunenburger, D. Lasseux, J. Petit, and J.-P. Delville, Eddies and interface deformations induced by optical streaming, J. Fluid Mech. 688, 195 (2011).

[8] J. Lighthill, Acoustic streaming, J. Sound Vib. 61, 391 (1978).

[9] C. M. Poindexter, P. J. Rusello, and E. A. Variano, Acoustic Doppler velocimeter-induced acoustic streaming and its implications for measurement, Exp. Fluids 50, 1429 (2011).

[10] C. Poelma, Ultrasound imaging velocimetry: A review, Exp. Fluids 58, 3 (2017).

[11] A. Green, J. S. Marshall, D. Ma, and J. Wu, Acoustic streaming and thermal instability of flow generated by ultrasound in a cylindrical container, Phys. Fluids 28, 104105 (2016).

[12] J. Klima, Application of ultrasound in electrochemistry. An overview of mechanisms and design of experimental arrangement, Ultrasonics 51, 202 (2011).

[13] A. Mandroyan, M. L. Doche, J. Y. Hihn, R. Viennet, Y. Bailly, and L. Simonin, Modification of the ultrasound induced activity by the presence of an electrode in a sono-reactor working at two low frequencies (20 and $40 \mathrm{kHz}$ ). Part II: Mapping flow velocities by particle image velocimetry (PIV), Ultrason. Sonochem. 16, 97 (2009).

[14] M. C. Schenker, M. J. B. M. Pourquié, D. G. Eskin, and B. J. Boersma, PIV quantification of the flow induced by an ultrasonic horn and numerical modeling of the flow and related processing times, Ultrason. Sonochem. 20, 502 (2013).

[15] S. B. Barnett, G. R. Ter Haar, M. C. Ziskin, H.-D. Rott, F. A. Duck, and K. Maeda, International recommendations and guidelines for the safe use of diagnostic ultrasound in medicine, Ultrasound Med. Biol. 26, 355 (2000).

[16] J. A. Cosgrove, J. M. Buick, S. D. Pye, and C. A. Greated, PIV applied to Eckart streaming produced by a medical ultrasound transducer, Ultrasonics 39, 461 (2001). 
[17] J. Wu and G. Du, Acoustic streaming generated by a focused Gaussian beam and finite amplitude tonebursts, Ultrasound Med. Biol. 19, 167 (1993).

[18] G. Zauhar, H. C. Starritt, and F. A. Duck, Studies of acoustic streaming in biological fluids with an ultrasound Doppler technique, Brit. J. Radiol. 71, 297 (1998).

[19] M. C. Charrier-Mojtabi, A. Fontaine, and A. Mojtabi, Influence of acoustic streaming on thermo-diffusion in a binary mixture under microgravity, Int. J. Heat Mass Transfer 55, 5992 (2012).

[20] C. Suri, K. Takenaka, H. Yanagida, Y. Kojima, and K. Koyama, Chaotic mixing generated by acoustic streaming, Ultrasonics 40, 393 (2002).

[21] Y. K. Oh, S. H. Park, and Y. I. Cho, A study of the effect of ultrasonic vibrations on phase-change heat transfer, Int. J. Heat Mass Transfer 45, 4631 (2002).

[22] V. Frenkel, R. Gurka, A. Liberzon, U. Shavit, and E. Kimmel, Preliminary investigations of ultrasound induced acoustic streaming using particle image velocimetry, Ultrasonics 39, 153 (2001).

[23] W. Dridi, D. Henry, and H. Ben Hadid, Influence of acoustic streaming on the stability of melt flows in horizontal Bridgman configurations, J. Cryst. Growth 310, 1546 (2008).

[24] D. G. Eskin, Ultrasonic melt processing. Achievements and challenges, Mater. Sci. Forum 828-829, 112 (2015).

[25] V. Abramov, O. Abramov, V. Bulgakov, and F. Sommer, Solidification of aluminium alloys under ultrasonic irradiation using water-cooled resonator, Mater. Lett. 37, 27 (1998).

[26] J. S. Marshall and J. Wu, Acoustic streaming, fluid mixing, and particle transport by a Gaussian ultrasound beam in a cylindrical container, Phys. Fluids 27, 103601 (2015).

[27] B. Ubbenjans, C. Frank-Rotsch, J. Virbulis, B. Nacke, and P. Rudolph, Numerical analysis of the influence of ultrasonic vibration on crystallization processes, Cryst. Res. Technol. 47, 279 (2012).

[28] G. N. Kozhemyakin, L. V. Zolkina, and Y. Inatomi, Influence of ultrasound on crystal growth from solution and related flow visualization, Cryst. Growth Design 6, 2412 (2006).

[29] N. F. Declercq, J. Eisener, A. Lippert, T. Nowak, C. Cairós, F. Reuter, and R. Mettin, Characterization of acoustic streaming beyond $100 \mathrm{MHz}$ [Phys. Procedia 70, 151 (2015)] .

[30] H. Ben Hadid, W. Dridi, V. Botton, B. Moudjed, and D. Henry, Instabilities in the Rayleigh-Bénard-Eckart problem, Phys. Rev. E 86, 016312 (2012).

[31] W. Dridi, D. Henry, and H. Ben Hadid, Influence of acoustic streaming on the stability of a laterally heated three-dimensional cavity, Phys. Rev. E 77, 046311 (2008).

[32] W. Dridi, D. Henry, and H. Ben Hadid, Stability of buoyant convection in a layer submitted to acoustic streaming, Phys. Rev. E 81, 056309 (2010).

[33] T. P. Lyubimova and R. V. Skuridin, Acoustic wave effect on a stability of convective flow in a horizontal channel subjected to the horizontal temperature gradient, Int. J. Heat Mass Transfer 104, 478 (2017).

[34] B. Moudjed, Caractérisation expérimentale et théorique des écoulements entraînés par ultrasons. Perspectives d'utilisation dans les procédés de solidification du silicium photovoltaïque, Ph.D. thesis, INSA de Lyon, 2013.

[35] B. Moudjed, V. Botton, D. Henry, S. Millet, J.-P. Garandet, and H. Ben Hadid, Oscillating acoustic streaming jet, Appl. Phys. Lett. 105, 184102 (2014).

[36] B. Moudjed, V. Botton, D. Henry, H. Ben Hadid, and J.-P. Garandet, Scaling and dimensional analysis of acoustic streaming jets, Phys. Fluids 26, 093602 (2014).

[37] B. Moudjed, V. Botton, D. Henry, S. Millet, J.-P. Garandet, and H. Ben Hadid, Near-field acoustic streaming jet, Phys. Rev. E 91, 033011 (2015).

[38] B. Moudjed, V. Botton, D. Henry, S. Millet, and H. Ben Hadid, Y-shaped jets driven by an ultrasonic beam

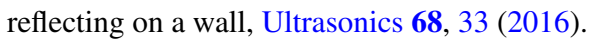

[39] R. Ben Haj Slama, B. Gilles, M. Ben Chiekh, and J.-C. Béra, PIV for the characterization of focused field induced acoustic streaming: seeding particle choice evaluation, Ultrasonics 76, 217 (2017).

[40] H. Ben Hadid and D. Henry, Numerical study of convection in the horizontal Bridgman configuration under the action of a constant magnetic field. Part 2. Three-dimensional flow, J. Fluid Mech. 333, 57 (1997).

[41] See Supplemental Material at http://link.aps.org/supplemental/10.1103/PhysRevFluids.2.123901 for movies. 
[42] J. Zhou, R. J. Adrian, S. Balachandar, and T. M. Kendall, Mechanisms for generating coherent packets of hairpin vortices in channel flow, J. Fluid Mech. 387, 353 (1999).

[43] T. Cambonie and J.-L. Aider, Transition scenario of the round jet in crossflow topology at low velocity ratios, Phys. Fluids 26, 084101 (2014).

[44] A. Bejan, Convection Heat Transfer (Wiley, New York, 2013).

[45] K. Hasegawa, L. Qiu, A. Noda, S. Inoue, and H. Shinoda, Electronically steerable ultrasound-driven long narrow air stream, Appl. Phys. Lett. 111, 064104 (2017).

[46] S. Dumitrica, D. Vizman, J.-P. Garandet, and A. Popescu, Numerical studies on a type of mechanical stirring in directional solidification method of multicrystalline silicon for photovoltaic applications, J. Cryst. Growth 360, 76 (2012).

[47] R. J. Moreau, Magnetohydrodynamics (Springer Science \& Business Media, New York, 2013), Vol. 3. 